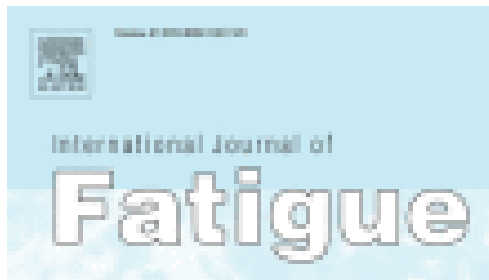

\section{Ia:}

Title: "An approximated methodology for fatigue tests and fatigue monitoring of concrete specimens"

Authors: A.J. Nieto, J.M. Chicharro y P. Pintado International Journal of Fatigue, 28 pp835-842 (2006)

Keywords:

Fatigue Tests, Accumulated Damage, Concrete, Damage Monitoring

doi:10.1016/j.ijfatigue.2005.11.004 


\title{
AN APPROXIMATED METHODOLOGY FOR FATIGUE TESTS AND FATIGUE MONITORING OF CONCRETE SPECIMENS
}

\author{
A.J.NIETO, J.M.CHICHARRO and P.PINTADO \\ Área de Ingeniería Mecánica \\ E.T.S.I.Industriales(Universidad de Castilla-La Mancha) \\ Avda. Camilo José Cela s/n, 13071-Ciudad Real (Spain) \\ e-mail: antoniojavier.nieto@uclm.es
}

November 11, 2005

\begin{abstract}
The determination of the number of cycles to failure for different stress amplitudes in concrete specimens, that is, the determination of the $\mathrm{S}-\mathrm{N}$ curve, is a process that could take an extremely long period of time if statistical significance is sought. This paper proposes a simplified approximated method that could be used to speed up the process and still obtain meaningful results. The method is based on assumptions with regard to the shape of the $\mathrm{S}-\mathrm{N}$ curve, and on assumptions related to damage accumulation and the influence of mean stress. As a second objective of this work, damage accumulation has been monitored in the specimens subjected to cyclic loads. The specimens have been monitored after each group of cycles. The evolution of the first resonance frequency as a function of number of cycles has been studied and the variation of the apparent Young's modulus of an equivalent Euler Bernoulli beam has also been determined. This modulus presents a decreasing zone up to a million of cycles, whereas no noticeable variations are found after this sharp decrease. This result could be useful in devising monitoring schemes for other type of structures.
\end{abstract}

Keywords: Fatigue Tests, Accumulated Damage, Concrete, Damage Monitoring PACS codes: 46.50.+a, 62.20.Mk, 81.70.Bt 


\section{Introduction}

Concrete is often used in structures subjected to cyclic loads. One such application is pavement infrastructure. In these cases, the fatigue behavior of the material needs to be characterized. The most basic information about the fatigue behavior of concrete specimens is represented by its $\mathrm{S}-\mathrm{N}$ curve, which provides the number of cycles to failure versus stress amplitude. The number of cycles to failure approaches 1 as the stress amplitude approaches the ultimate stress of concrete. The time required to carry out one such high stress fatigue test is certainly not a problem. Nevertheless, when the stress amplitude is small compared to the ultimate stress, the number of cycles to failure is very high, and days may easily be required to carry out just one test. Moreover, fatigue results are usually highly scattered, and a good number of tests are needed for each stress amplitude in order to obtain statistically meaningful results [1]. This is particularly true for concrete, a material whose structure and fabrication process are such that relatively large standard deviations are observed in its mechanical properties. Furthermore, concrete structures are always constructed with concrete fabricated in different batches, and sometimes even from different suppliers. Both factors contribute to an increase in the standard deviation of the ultimate stress and in the number of cycles to failure for a given stress amplitude.

Large scatter, along with prolonged testing times, make fatigue characterization a tedious process. This paper presents a new simplified approach that could be used to obtain a useful S-N curve. The method assumes that the unknown $\mathrm{S}-\mathrm{N}$ curve may be represented by a straight line in logarithmic scale. This assumption alone simplifies the process tremendously since the number of parameters to be determined is now reduced to just two. But even in this case, low stress fatigue tests may consume large amounts of time. In order to overcome this problem, all specimens in this work will be subjected to cycle groups of increasing amplitude. This method implies, on the one hand, that there will be certain areas of the S-N map that will not be explored, which is a drawback; but on the other hand it implies that all specimens will fail before the number of cycles, and thus, time becomes too large, which is an advantage for a simplified method as the one sought in this study.

A second objective of this work is to present the correlation between the vibration response of the specimen and the accumulated damage. This is a subject that has received considerable attention in the past few decades. The subject has been addressed from many different angles. For example, Kosmatka and Ricles [2] investigated stiffness variations as damage is being inflicted on the specimen; Cawley and Ray [3] studied the influence of cracks and slots on specimen eigenfrequencies; Armon et. al. [4] considered using rank-ordering of eigenfrequency shifts as a method for crack detection; Vurpillot et. al. used fiber optic deformation sensors to monitor bridges [5]; and Sebaaly et. al. considered pavement monitoring by dynamically interpreting the results obtained with the ubiquous Falling Weight Deflectometer [6].

Our work investigates the variation of eigenfrequencies with accumulated damage. This variation was 
also studied by Popovics et. al. using concrete cylinders [7]. Our experimental setup is similar, the difference being the shape and size of the specimens. Each concrete specimen is loaded at one end using an impact hammer, and its vibrational response is captured by an accelerometer at the opposite end. This process is repeated after the specimen has been subjected to each cycle group, that is, after damage has been inflicted on the specimen.

\section{Methodology}

The method for accelerating the determination of the S-N curve is based on a few assumptions. The first assumption is that the unknown S-N curve may be represented by a straight line in logarithmic scale [8]:

$$
S=\sigma_{u} \cdot N^{b}
$$

where $N$ is the number of cycles to failure, $\sigma_{u}$ is the ultimate stress of the material, and $b$ is the straight line slope.

This assumption simplifies the process tremendously since the number of parameters to be determined is now reduced to just two: $\sigma_{u}$ and $b$. The ultimate stress of the material, $\sigma_{u}$, may be obtained from a series of static tests. In this work, prismatic specimens and a four point bending rig are used for the static tests as well as for the fatigue tests. Details of the experimental setup will be discussed in Section 3. A number of specimens are statically loaded up to failure in order to determine a meaningful ultimate stress average $\sigma_{u}$.

Dynamic tests are performed in order to determine the slope parameter $b$. Nevertheless, it was pointed out in the previous section that low stress fatigue tests may consume large amounts of time, and that to overcome this problem, all specimens in this work will be subjected to cycle groups of increasing amplitude. In an ideal world, all specimens would fail in the same cycle group and after the same number of cycles. The method would provide just one data point in the $\mathrm{S}-\mathrm{N}$ curve. Nevertheless, this single point would suffice to determine the slope parameter $b$. But fatigue behavior is far from being ideal and, on the contrary, the results are usually highly scattered. This means that subjecting the specimens to cycle groups of increasing amplitude will permit to be detected the weak specimens that fail in the first low amplitude cycle groups, as well as the strong specimens that fail after the large amplitude cycle groups are applied. There are certain areas of the $\mathrm{S}-\mathrm{N}$ map that are not explored. The high amplitude-low cycle area is not investigated, and the low amplitude-high cycle is avoided in order to speed up the determination of the S-N curve. Nonetheless, a statistically significant number of specimens could be tested in this manner to obtain a meaningful value of the slope $b$. 
The translation of the test results to the $\mathrm{S}-\mathrm{N}$ map requires that the following two factors be taken into account: damage accumulation and average stress. The latter is a consequence of the test setup. The four point bending rig (Fig. 1) applies bending moments that fluctuate from zero to a maximum value. No alternating bending moment may be applied and, therefore, the average stress is not zero. Several models have been proposed to take into account the influence of average stress. Nonlinear models, such as those by Gerber, Kececioglu, or Bagci, have been discarded in favor of the simpler Goodman linear model:

$$
\frac{\sigma_{a}}{S_{e}}+\frac{\sigma_{m}}{\sigma_{u}}=1
$$

where $\sigma_{a}$ is the amplitude of the fluctuating stress, $\sigma_{m}$ is the average stress, and $S$ is the fatigue limit to be translated to the $\mathrm{S}-\mathrm{N}$ plot.

The accumulation of damage also needs to be modelled to take into account the different amplitude cycle groups. In this case, the simple Palmgren-Miner principle has been used. The Palmgren-Miner rule is not very accurate for concrete specimens. Some authors have investigated improved damage accumulation rules for concrete fatigue tests (see for example [9] and [10]). Nevertheless, the loss of accuracy is acceptable when one looks for a simple and fast process to obtain $\mathrm{S}-\mathrm{N}$ curves. The S-N curve shown in this paper has been obtained assuming that the Palmgren-Miner rule is valid. However, the results compare well with those obtained by other authors using methods that did not require the assumption of a rule for damage accumulation (see, for example [11] and [12]). According to this theory, the damage inflicted by a single cycle is inversely proportional to the number of cycles of the same amplitude that would make the specimen fail. Therefore, the damage $D_{i}$ inflicted by a group of $n_{i}$ cycles all of the same amplitude is:

$$
D_{i}=\frac{n_{i}}{N_{i}}
$$

where $N_{i}$ is the total number of cycles of the same amplitude that the specimen would take before failure. If $n_{i}=N_{i}$, then $D_{i}=1(100 \%)$, and the specimen fails. The principle allows for the estimation of the damage accumulated when different amplitude cycles are applied to the specimen. In this case, the specimen fails when the accumulated damage reaches $100 \%$ :

$$
D=\sum_{i=1}^{i=k} D_{i}=1
$$

$k$ being the number of different cycle groups.

Combining equations 1,2 and 3, one may express the damage $D_{i}$ caused by the $n_{i}$ cycles of group $i$ in terms of the average and alternating stresses in this cycle group, $\sigma_{i}^{m}$ and $\sigma_{i}^{a}$, and in terms of the material parameters, $\sigma_{u}$ and $b$, as follows:

$$
D_{i}=n_{i}\left(\frac{\sigma_{u}-\sigma_{i}^{m}}{\sigma_{i}^{a}}\right)^{\frac{1}{b}}
$$


When the damage accumulated reaches $100 \%$, that is, when:

$$
1=\sum_{i=1}^{i=k} n_{i}\left(\frac{\sigma_{u}-\sigma_{i}^{m}}{\sigma_{i}^{a}}\right)^{\frac{1}{b}}
$$

the specimen fails. Each specimen fails in a different cycle group and after a different number of cycles. Therefore, the previous expression provides different values of $b$ for each fatigue test. The average value could be used to determine a useful S-N curve.

It was mentioned that a second objective of this work is to investigate the variation of eigenfrequencies with accumulated damage. Eigenfrequencies are determined from the Fourier transform of the transfer function obtained when measuring acceleration after an impact force is applied to the specimen. The lowest frequency in this transfer function should correspond to the first bending mode of the concrete beam. This fact may be used to obtain an estimation of the Young's modulus of the material. Although the estimation may not be very accurate, it is useful to determine apparent Young's modulus variation with accumulated damage.

The vibration response of the specimen changes as cracks develop in the material. The first resonance peak of the damaged beam may be used to define an apparent Young's modulus as follows: the apparent Young's modulus is the elastic modulus of a theoretical (and undamaged) Euler-Bernoulli beam whose first resonance frequency coincides with that of the real (and damaged) beam.

If Euler-Bernoulli beam theory is used, the bending vibration of the beam is described by the following equation:

$$
\frac{\partial^{2} u}{\partial t^{2}}+c^{2} \frac{\partial^{4} u}{\partial x^{4}}=0
$$

where $u$ is the transversal displacement of the neutral axis of the beam, and $c=\sqrt{E I / \rho A}$ is the flexural wave propagation velocity expressed in terms of the Young modulus of the material $E$, its density $\rho$, the cross section moment of inertia $I$ and its area $A$. The previous equation provides the flexural eigenfrequencies, the lowest of which, disregarding the rigid body motion null frequency, is:

$$
\omega_{1}=\left(\beta_{1}\right)^{2} \sqrt{\frac{E I}{A \rho}}
$$

where $\beta_{1} L=4.730041$ and $L$ is the length of the specimen [13]. The equation can be used to obtain the Young's modulus as a function of the measured frequency:

$$
E=\left(\frac{2 \pi f_{1}}{\left(\beta_{1}\right)^{2}}\right)^{2} \frac{A \rho}{I}
$$

The specimens used in this study are short beams, thus, the Euler-Bernoulli beam model is just a rough approximation of their bending behavior. Moreover, the material density has not been accurately 
determined in this work. Therefore, this estimation of the Young's modulus is not very accurate. Nevertheless, the estimation is useful for determine variations of the apparent modulus with damage. The modulus obtained in this manner is an apparent modulus in the sense that it corresponds to the ideal Euler-Bernoulli beam without a notch and without fatigue cracks.

\section{$3 \quad$ Experimental test setup}

Figure 2 shows a diagram of the test procedure. The specimens are subjected to four-point-bending using the load rig shown in Figure 1. Fatigue tests are carried out on an MTS Model 810 fatigue test machine using load as the control parameter. The four-point-bending rig has been built with stiff steel beams (IPE 180 [14]) so that its deflection can be neglected when compared to that of the specimen. The two upper load points are $132 \mathrm{~mm}$ apart $\left(d_{1}=132 \mathrm{~mm}\right.$, see Fig. 3(a)), whereas the two lower load points are $355 \mathrm{~mm}$ apart $\left(d_{2}=355 \mathrm{~mm}\right.$, Fig. $\left.3(\mathrm{a})\right)$.

The concrete specimens used in this work are $406 \mathrm{~mm}$ long, $76 \mathrm{~mm}$ wide, and $76 \mathrm{~mm}$ high. A $5 \mathrm{~mm}$ notch has been created on the tension side of each specimen. When the test machine applies a force $F$, the nominal stress on the bottom face of the specimen, disregarding the stress concentration created by the notch, is:

$$
\sigma=F\left(\frac{3 d}{b h^{2}}\right)
$$

where $d$ is the distance between forces in each couple $d=\left(d_{2}-d_{1}\right) / 2$.

Expression 10 is an indirect stress measurement. The evaluation of uncertainty complies with the ISO "Guide to the Expression of Uncertainty in Measurement" [15]. Specimen geometrical parameters (width and hight) have been obtained with a $0.05 \mathrm{~mm}$ precision digital caliper. But, on the other hand, the load rig support spacing had to be measured using a measuring tape with a $1 \mathrm{~mm}$ resolution. The main contributor to load uncertainty is hydraulic actuator calibration $(u(F)=2 \mathrm{~N})$. Therefore, the expanded uncertainty of measurement of stress due to testing method is $U(\sigma)=0.085 \mathrm{MPa}$, with a coverage factor $k=2$, which for a normal distribution corresponds to a coverage probability of approximately $95 \%$.

Static tests are performed to obtain the ultimate material stress $\sigma_{u}$. The pseudo-static conditions are achieved by increasing the load at a very slow rate of $1 \mathrm{kN}$ in 60 seconds. It has been mentioned that relatively large standard deviations are observed in the mechanical properties of concrete, but a normality test has been conducted to guarantee that the resulting average $\bar{\sigma}_{u}$ and standard deviation $u\left(\sigma_{u}\right)$ characterize the statistical distribution of the specimen population.

Dynamic tests are performed to obtain the $\mathrm{S}-\mathrm{N}$ slope parameter $b$. The test pattern is shown in 
Table 1. According to this table, the first 250000 cycles exert a maximum tension stress of $2.84 \mathrm{MPa}$ (mean stress $\sigma_{m}^{1}=1.61 \mathrm{MPa}$, and alternating stress $\sigma_{a}^{1}=1.23 \mathrm{MPa}$ ) which corresponds to $50 \%$ of the average ultimate stress $\sigma_{u}$. If the specimen hasn't failed after this first cycle group, then the maximum stress is increased to $55 \%$ of the average ultimate stress, and a second cycle group is started. The process continues, if necessary, following the cycle groups represented in Table 1. The load variation is sinusoidal, the frequency being $12 \mathrm{~Hz}$. Some authors have chosen different frequencies for their fatigue tests. For example, Zhang et al. [11] carried out their tests with a frequency of $4.5 \mathrm{~Hz}$ and Murdock et al. [12] with a frequency of $6-7 \mathrm{~Hz}$. Provided that the frequency is not too high, its value is commonly considered to not have a great influence on fatigue results. Mean stress is always slightly larger than alternating stress because the contact load between the specimen and the load rig should not go down to zero. A minimum compressive load is kept in order to avoid possible undesired displacements of the specimen with respect to the load rig.

The temperature and relative humidity were not controlled precisely. Nevertheless, both fatigue and monitoring tests have been carried out in an air conditioned laboratory where temperature and relative humidity values did not undergo significant fluctuations. The controlled values for temperature and relative humidity were $20 \pm 2^{\circ} \mathrm{C}$ and $50 \%$ respectively.

Damage has been monitored by impacting the specimens on the upper face center point and measuring acceleration on the same face at a distance of a quarter length from the impact point. The specimen is supported on a flexible foam bed during the test. The piezoelectric accelerometers (B\&K 4371) cover a range of frequencies from 0.1 to $12600 \mathrm{~Hz}$, the nominal voltage acceleration sensitivity being 1.02 $\mathrm{mV} /\left(\mathrm{m} / \mathrm{s}^{-2}\right)$. The impact hammer (B\&K 8202) mass is $280 \mathrm{~g}$, generating a pulse with a duration range from 0.2 to $0.25 \mathrm{~ms}$. A trigger is used to synchronize the acquisition of force and acceleration signals. Both signals are logged in the computer at a sampling rate of 12.6 ksamples/s using a data acquisition card PCI-MIO-16E-1 from National Instruments. Each signal is represented with a total of 1024 data points. These two signals allow for the calculation of the Transfer Function FFT. The objective is to determine the variation of the first resonance peak of this transfer function FFT as damage is being inflicted on the specimen. This is done after every cycle group is completed. Figure 4 shows a typical acceleration time signal. The corresponding transfer function Fourier transform (FFT) is shown in Figure 5.

The fact that this resonance peak corresponds to the first mode of bending motion of the free beam has been checked by comparing an indirect estimation of the Young's modulus with that obtained through eq. 9 using the measured frequency. According to [16], the Young's modulus of concrete is directly related to its compression strength $f_{c m}$ through the equation $E=10000 \sqrt[3]{f_{c m}}$, where $f_{c m}$ is the compression strength in $\mathrm{N} / \mathrm{mm}^{2}$. The nominal strength for a concrete mixture as that used in this study is $25 \mathrm{~N} / \mathrm{mm}^{2}$, therefore, the estimated Young's modulus is $E_{T}=29.24 \mathrm{GPa}$. On the other hand, a sample undamaged 
specimen provided a lowest frequency of $1594 \mathrm{~Hz}$ which corresponds to an elastic modulus of $27.77 \mathrm{GPa}$ according to eq. 9. The two values of the Young modulus obtained as explained in this paragraph do not differ more than $5 \%$, and this allows us to affirm that the lowest resonance peak corresponds to the first mode of bending motion.

Figure 5 shows the quotient between the Fourier Transforms of both excitation and response. The peaks in this Transfer Function correspond to the specimen eigenfrequencies. The first eigenfrequency is used to evaluate the Young's modulus. The uncertainty in this elastic modulus is influenced by the uncertainty in the determination of the first eigenfrequency, the geometrical dimensions, and mass. Each vibration test was repeated three times and the eigenfrequency was taken as the average of the three eigenfrequency values obtained in all three measurements. The maximum variation between these three measures in all the tests carried out in this study was $15 \mathrm{~Hz}$. Therefore, the maximum uncertainty in the elastic modulus is $0.31 \mathrm{GPa}$, which corresponds to a relative uncertainty of $1.1 \%$.

\section{Experimental results}

The material used for static and fatigue tests was provided by two different local suppliers. The nominal compression strength is $25 \mathrm{~N} / \mathrm{mm}^{2}$, and the aggregate nominal maximum size is $20 \mathrm{~mm}$. The water/cement ratio is 0.6 , and the cement dosage is $315 \mathrm{~kg} / \mathrm{m}^{3}$. The cement used is CEM II/A-M 42,5 R [17]. The specimens were molded in a prismatic mold of appropriate size from which they were extracted after 48 hours. The specimens were stored under controlled temperature $\left(20^{\circ} \mathrm{C}\right)$ and controlled relative humidity (95\%) for 28 days.

Fifty specimens were tested under quasi-static load. Force measurements are translated into nominal stress using eq. 10. The probability distribution of the ultimate nominal stress has been depicted in Fig.6 where a normal fit has also been plotted. These results provide an average value of $\bar{\sigma}_{u}=5.68 \mathrm{MPa}$ and a standard deviation of $u\left(\sigma_{u}\right)=0.891 \mathrm{MPa}$, which represents a coefficient of variation of $15.7 \%$. This coefficient of variation is not high for a population that has been provided by two different suppliers. The samples have not been fabricated under controlled laboratory conditions but, rather, have been produced in an industrial plant in an effort to simulate the actual scatter found in concrete infrastructures.

A normal distribution is a valid fit for the obtained experimental results. This statement has been proven by applying the Shapiro and Wilks [18] criteria to the ultimate stress data. The test is valid for the 50 sample size population and is significant at the $5 \%$ level.

Ten specimens were tested under cyclic load. The total number of cycles to failure for each specimen is shown in Fig.7. As was described in the previous section, these cycles are grouped in batches of 250000 
cycles, the load amplitude of which increase from batch to batch. The frequency response of the specimen is tested before the next cycle group is applied. It was also explained in the previous section that each specimen provides, from equation 6 , a value for the slope parameter $b$. The average value obtained is $\bar{b}=-0.035$, whereas the standard deviation is $u(b)=0.015$.

It may be said that fatigue tests are usually highly scattered, but this statement is aggravated in the case of concrete and, particularly, in the case of concrete provided by several suppliers under standard construction conditions. This may be seen in Figure 8 where the resulting S-N curve has been plotted. The three curves in the figure correspond to the average value of $b$, as well as to the value one standard deviation above $(\bar{b}+u(b))$ and one standard deviation below $(\bar{b}-u(b))$. The stress axis in this plot is normalized using the static average ultimate stress.

The large scatter in fatigue results should not be attributed to the use of a simplified damage accumulation rule since this scatter is also present in the static strength tests. The reason for this scatter is the use of commercially available concrete with no additional control over its composition other than that performed routinely in mixing plants. The idea was to simulate real construction conditions.

As can be seen in the results of this work and using the explained methodology, one concrete specimen needs a mean of $1.7 \cdot 10^{6}$ cycles to failure. When a frequency rate of $12 \mathrm{~Hz}$ is used, 39 hours are needed on average to make one specimen fail and thus 390 hours to complete all 10 specimen tests. This time is compared to an estimation of the time required by other authors in Table 2. In order to appropriately compare the results, the following assumptions are made: first of all, $12 \mathrm{~Hz}$ frequency rate has been assumed in all cases; on the other hand, a few tests where artificially added to their curves in order to make the number of specimens consistent.

Damage has been monitored by measuring the first resonant peak in the transfer function as was explained in the previous section. This peak was measured in each undamaged specimen before applying any load, obtaining a frequency value that will be referred to as $f_{1 T}^{0}$, and subsequently after each cycle group has been completed, obtaining a frequency value that will be referred to as $f_{1 T}^{i}$, where $i$ is the number of cycles. Figure 9 shows the normalized frequency variation $\left(\Delta f=\left(f_{1 T}^{i}-f_{1 T}^{0}\right) 100 / f_{1 T}^{0}\right)$ as a function of the number of cycles. The results corresponding to the first cycle groups include all specimens since none had failed at such low number of cycles. The segments in Figure 9 represent the lowest, average, and highest values of normalized frequency variation. As the number of cycles increases, some specimens fail and the segments in Figure 9 describe a population with a lower number of specimens.

The normalized frequency variation results have been interpolated by the equation:

$$
\Delta f=-5.13 \cdot 10^{-10} x^{3}+2.61 \cdot 10^{-6} x^{2}-0.45 \cdot 10^{-2} x
$$


where $x$ is the number of cycles expressed in thousands of cycles (kcycle). This interpolating curve is also shown in Figure 9. The curve shows that the first resonant frequency decreases appreciably during the first million cycles, but remains almost constant hereafter.

Figure 10 shows the elastic modulus obtained from eq. 9 (using the measured eigenfrequency) as a function of the number of cycles. The segments in Figure 10 represent the lowest, average, and highest values of elastic modulus. These results have been interpolated by the equation:

$$
E=-1.03 \cdot 10^{-9} x^{3}+5.21 \cdot 10^{-6} x^{2}-8.94 x+27.71 \cdot 10^{+9}
$$

where, $x$ is the number of cycles. Figure 11 shows the normalized variation of elastic modulus, defined as $\Delta E=\left(E^{i}-E^{0}\right) 100 / E^{0}$, where $E^{0}$ is the modulus of the undamaged specimen, and $E^{i}$ is the modulus of the same specimen after $i$ cycles, versus the normalized fatigue life as was defined in eq. 5 and 6 . The figure may be divided in two zones, one in which the modulus decreases rapidly, and another in which the modulus remains almost constant. This result has also been observed by other researchers [19].

\section{Conclusions}

A simplified approximated methodology that could be used to speed up the determination of the S-N curve for concrete has been presented. The fatigue results obtained in this approximated manner are qualitatively similar to those obtained by other authors using different testing approaches [11], [12] and $[20]$.

This method minimizes the usually large efforts needed for this task. A mean of 390 hours are needed when this methodology and 10 specimens are used. This elapsed time represents half of the time required by other authors.

Damage accumulation has been monitored in the specimens subjected to cyclic loads. The variation of the first resonance frequency with respect to damage accumulation has also been studied. A distinctive correlation has been observed between damage and the first resonance frequency of the concrete specimens. These results are in agreement with those obtained by other authors [21]. There are clearly two regions in the plot of modulus versus normalized fatigue life. The first zone extends up to $40 \%$ of the normalized fatigue life, whereas from this point to failure the modulus remained almost constant. 


\section{Acknowledgment}

The authors would like to acknowledge the advice given by Prof. Alfonso Moraño.

\section{References}

[1] D. Kachlakev, S. Yim, T. Miller. "Behaviour of FRP composite-strengthened beams under static and cyclic loading”. Oregon Department of Transportation Research Group. June (2001).

[2] J.B. Kosmatka, J.M. Ricles. "Damage detection in structures by modal vibration characterization". Journal of Structural Engineering. December (1999).

[3] P. Cawley, R. Ray. "A comparison of the natural frequency changes produced by cracks and slots". Transactions of the ASME 100, 366-370. (1988).

[4] D. Armon, Y. Ben-Haim, S. Braun. "Crack detection in beams by rank-ordering of eigenfrequency shifts". Mechanical Systems and Signal Processing, 8(1), 81-91. (1994).

[5] S. Vurpillot, D. Inaudi, J-M. Ducret. "Bridge monitoring by fiber optic deformation sensors: design, emplacement and results". SPIE, Smart Structures and Materials. San Diego, USA. (1996).

[6] E. Sebaaly, S. Mamlouck, T. Davies. "Dynamic Analysis of falling weight deflectometre data", Transportation Research Record 1070, pp 63-78. (1986).

[7] J.S. Popovics, K.V. Subramaniam, S.P. Shah. "Vibrational resonances in finite length concrete cylinders". Proceedings of the ASNT's Spring Conference Sixth Annual Research Symposium, March 17-21, Houston (1997).

[8] S.Suresh, "Fatigue of Materials". Cambridge University Press, Second Edition (1998).

[9] B.H. Oh. "Cumulative damage theory of concrete under variable-amplitude fatigue loadings". ACI Materials Journal. 1991(88), No.1. 41-48.

[10] H.K. Hilsdorf and C.E. Kesler. "Fatigue strength of concrete under varying flexural stresses". Journal of the American concrete Institute, 1966(10),1059-1076.

[11] J. Zhang, H. Stang. "Fatigue performance in flexure of fiber reinforced concrete". ACI Mat. J. 95(1), 58-67. (1998).

[12] J.W. Murdock, and C.E. Kesler. "Effect of range of stress on fatigue strength of plain concrete beams". ACI J., 55 (2), 221-232.(1959)

[13] S.Rao, "Mechanical vibrations".Addison-Wesley Publishing Company, Third Edition (1995). 
[14] Norma Básica de la Edificación NBE EA-95. "Estructuras de acero en edificación". Real Decreto 1829/1995 de 10 de noviembre. Publicado en el Boletín Oficial del Estado de 18 de Enero de 1996.

[15] ISO (International Organization for Standardization), "Guide to the expresion of uncertainy in measurement", Switzerland(1995)

[16] EHE. "Instrucción de hormigón estructural". ICCE (Intituciones Colegiales para la Calidad en la Edificación). 1ª Edición. Leynfor Siglo XXI. Mayo (1999).

[17] UNE-EN 197-1. "Cement. Part 1: Composition, specifications and conformity criteria for common cements". December (2000).

[18] G.E.P. Box, W.G. Hunter, J.S. Hunter. "Statistics for Experimenters". John Wiley and Sons (1993).

[19] K.V. Subramaniam, J.S. Popovics, S.P. Shah. "Monitoring fatigue damage in concrete". Nondestructive Characterization of Materials in Aging Systems MRS Symposium Proceedings Series, pp. 151-157, Volume 503, Material Research Society, Warrendale, PA, (1998).

[20] J. Zhang, V.C. Li, H. Stang. "Size effect on fatigue in bending of concrete". Journal of Materials in Civil Engineering, November (2001).

[21] A.C. Collop, D. Cebon. "Stiffness reductions of flexible pavements due to cumulative fatigue damage". Journal of Transportation Engineering, p.131-139, March/April (1996).

[22] E. Kohler, A. Ali and J. Harvey. "Flexural fatigue life of hydraulic cement concrete beams". California Department of Transportation. University of California. (2005). 


\section{Figure captions}

Figure 1. Fatigue test load rig.

Figure 2. Test procedure schematics.

Figure 3. a) Four-point-bending layout. b) Bending moment diagram.

Figure 4. Accelerometer response.

Figure 5. Transfer function.

Figure 6. Probability density function of the ultimate stress (S) and the normal distribution that fit the data.

Figure 7. Distribution of the number of cycles to failure.

Figure 8. Normalized stress versus number of cycles.

Figure 9.Normalized frequency variation versus number of cycles.

Figure 10.Young's modulus versus number of cycles.

Figure 11. Normalized variation of elastic modulus versus normalized fatigue life.

\section{Tables}

Table 1. Dynamic test pattern.

Table 2. Time required for other authors' conventional tests (References: ${ }^{(*)}$ E.Kholer [22]) 


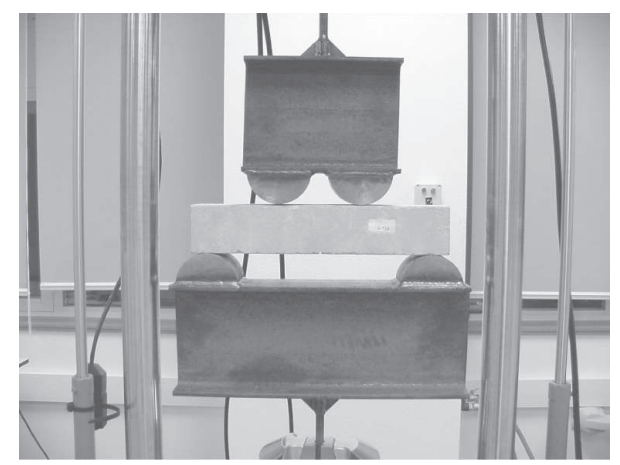

Figure 1: Fatigue test load rig.

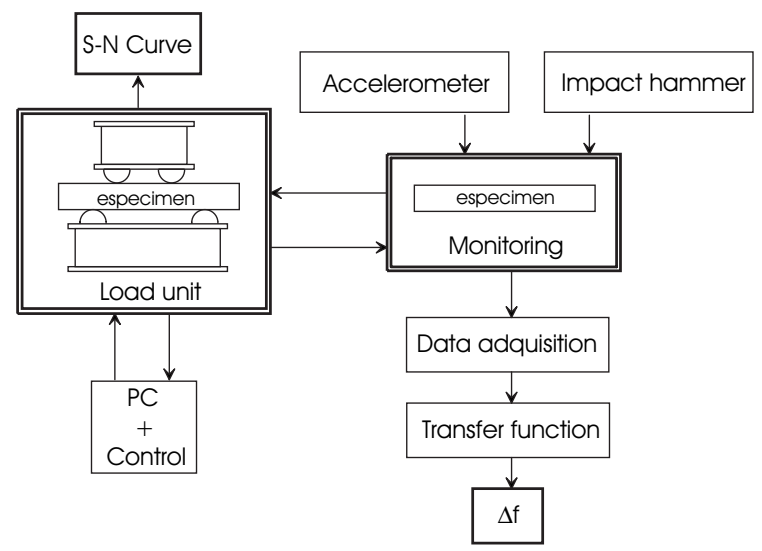

Figure 2: Test procedure schematics.

a)
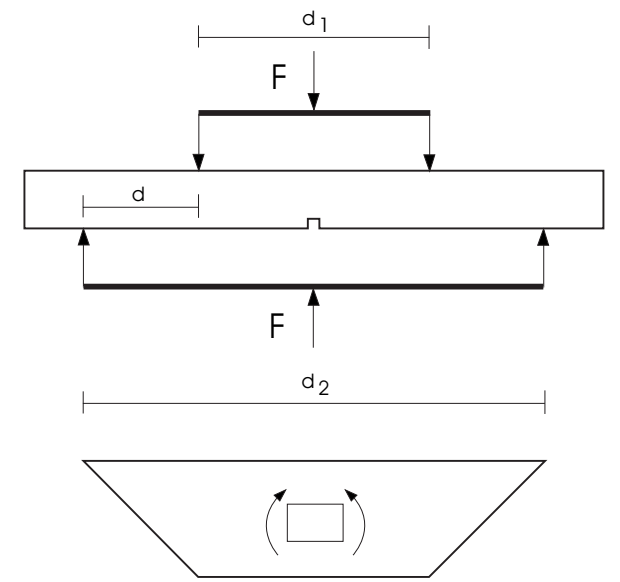

Figure 3: a) Four-point-bending layout. b) Bending moment diagram. 


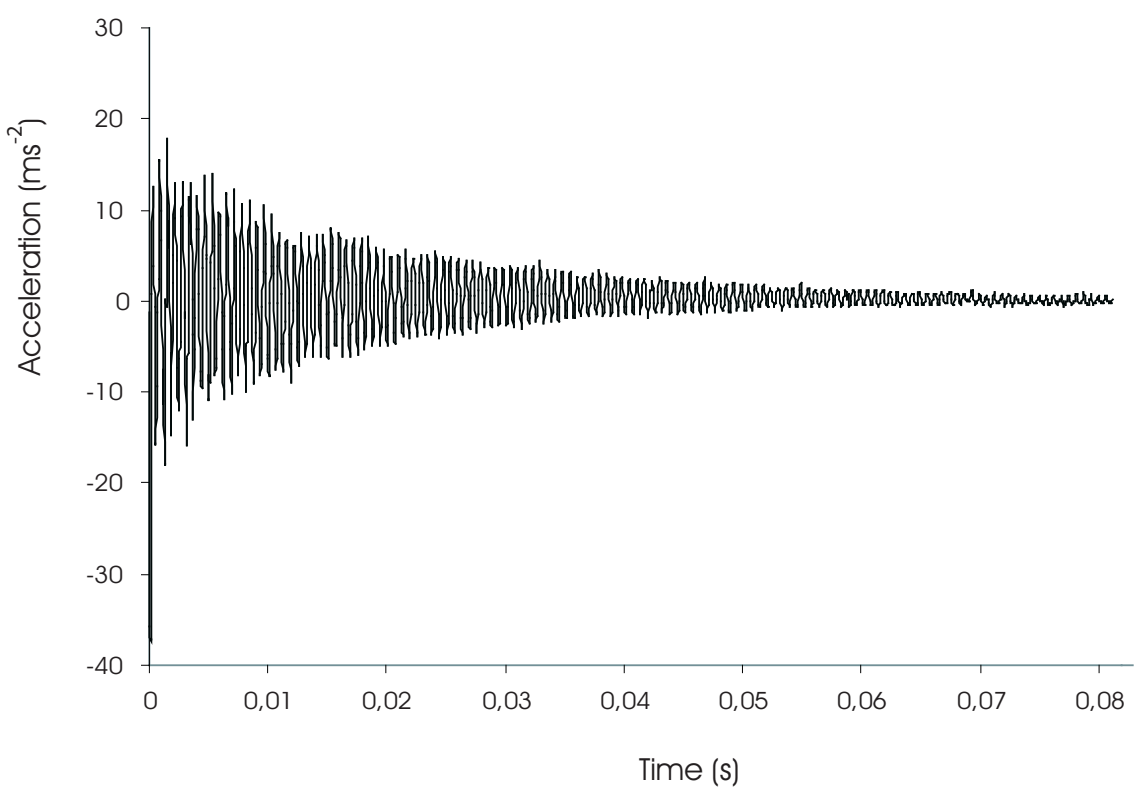

Figure 4: Accelerometer response.

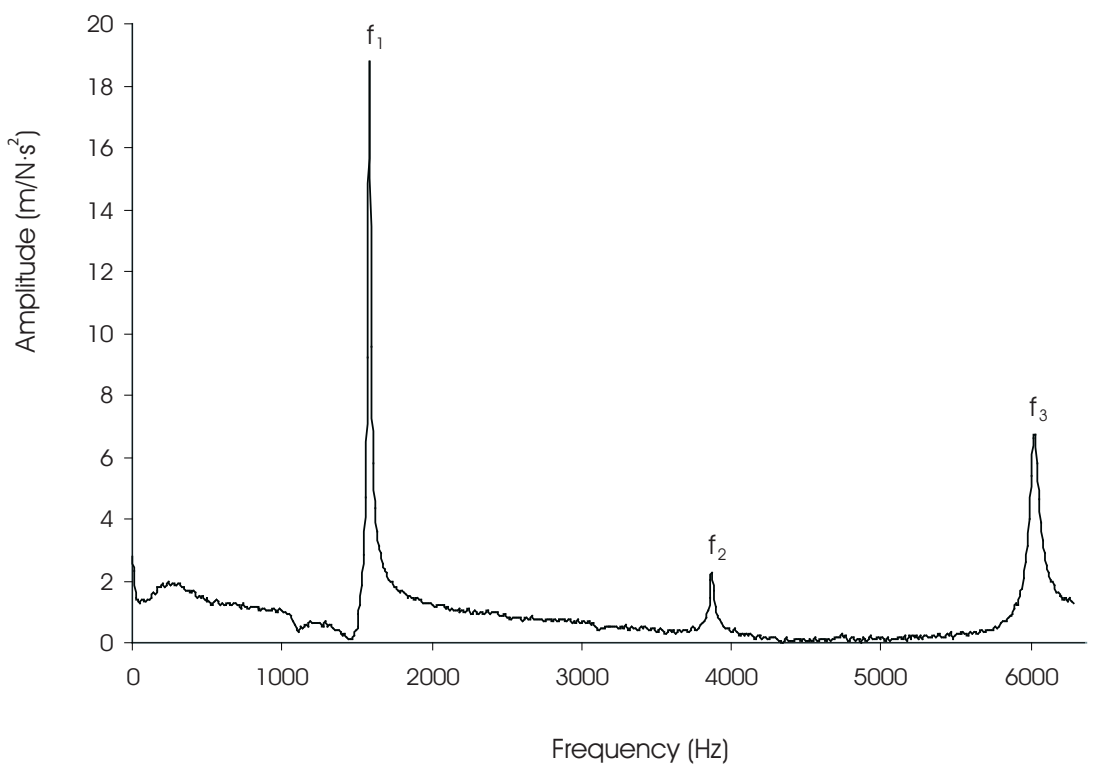

Figure 5: Transfer function. 


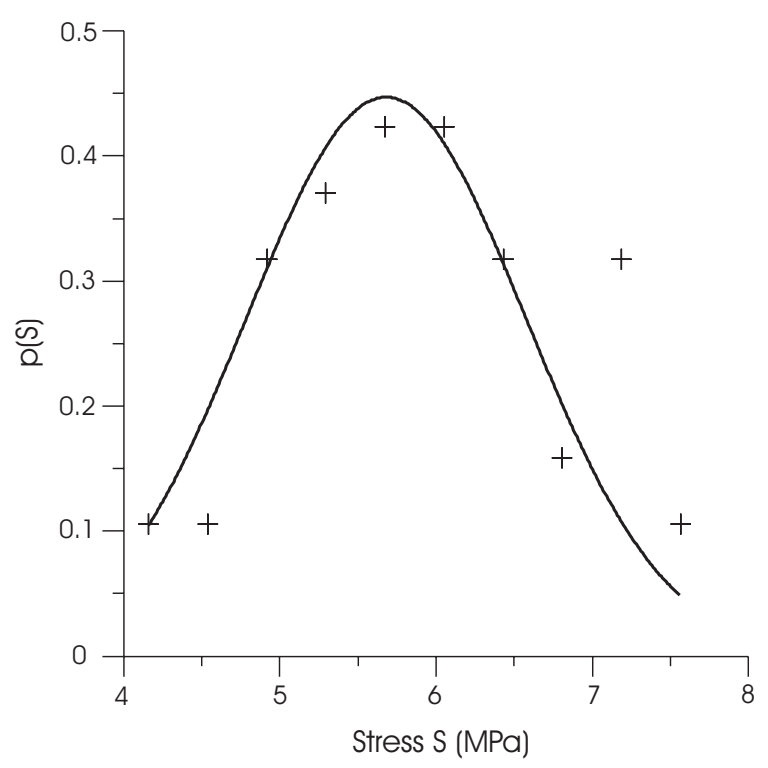

Figure 6: Probability density function of the ultimate stress (S) and the normal distribution that fit the data.

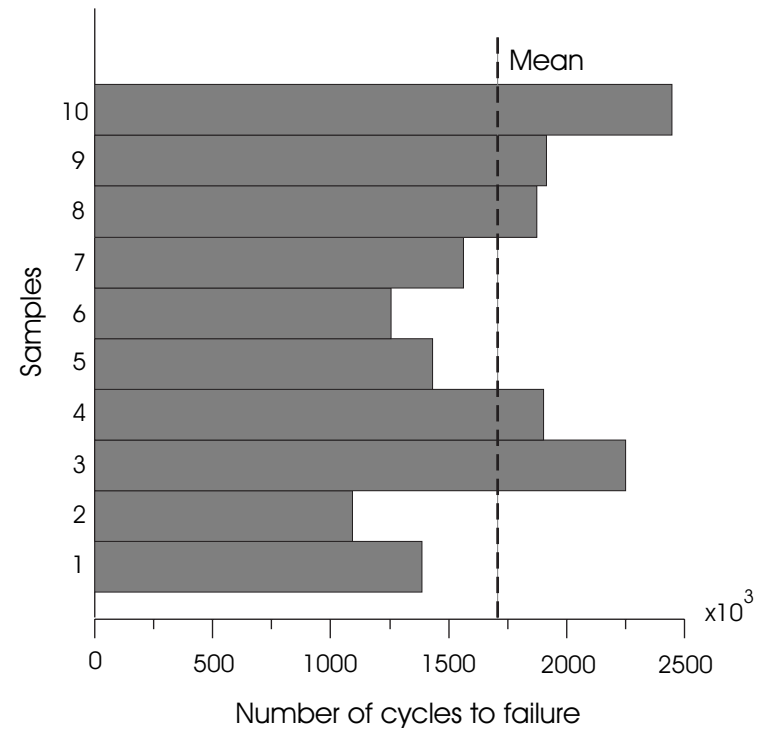

Figure 7: Distribution of the number of cycles to failure. 


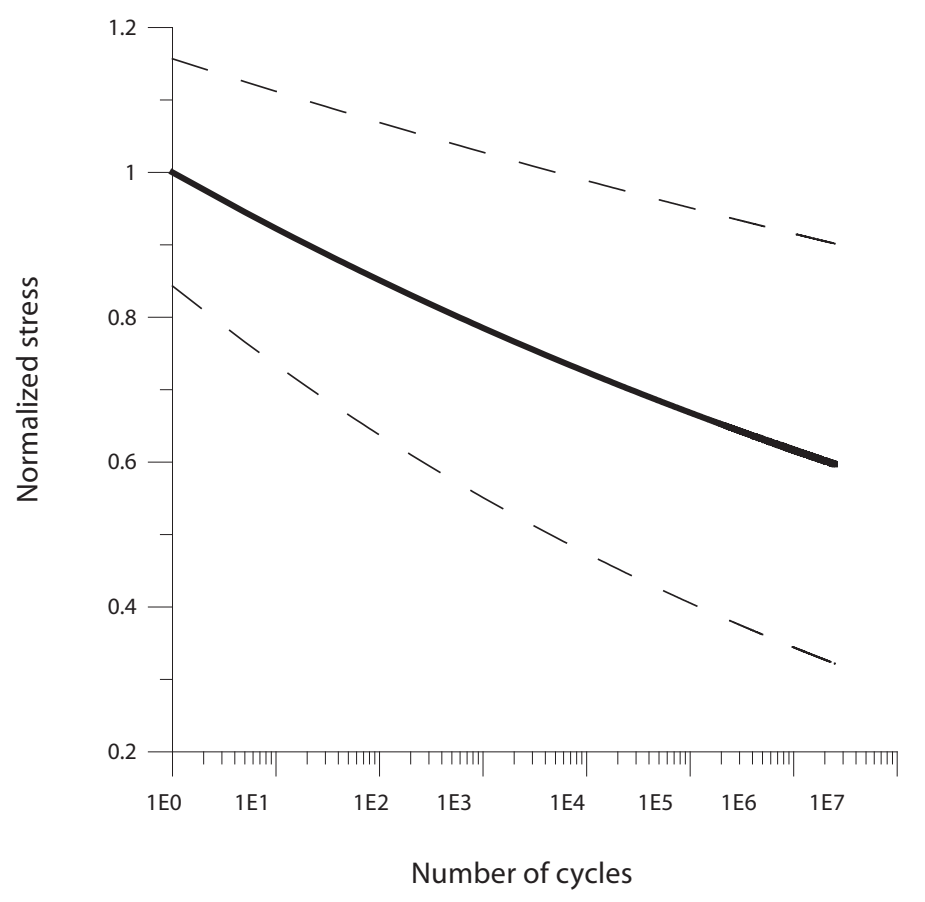

Figure 8: Normalized stress versus number of cycles to failure.

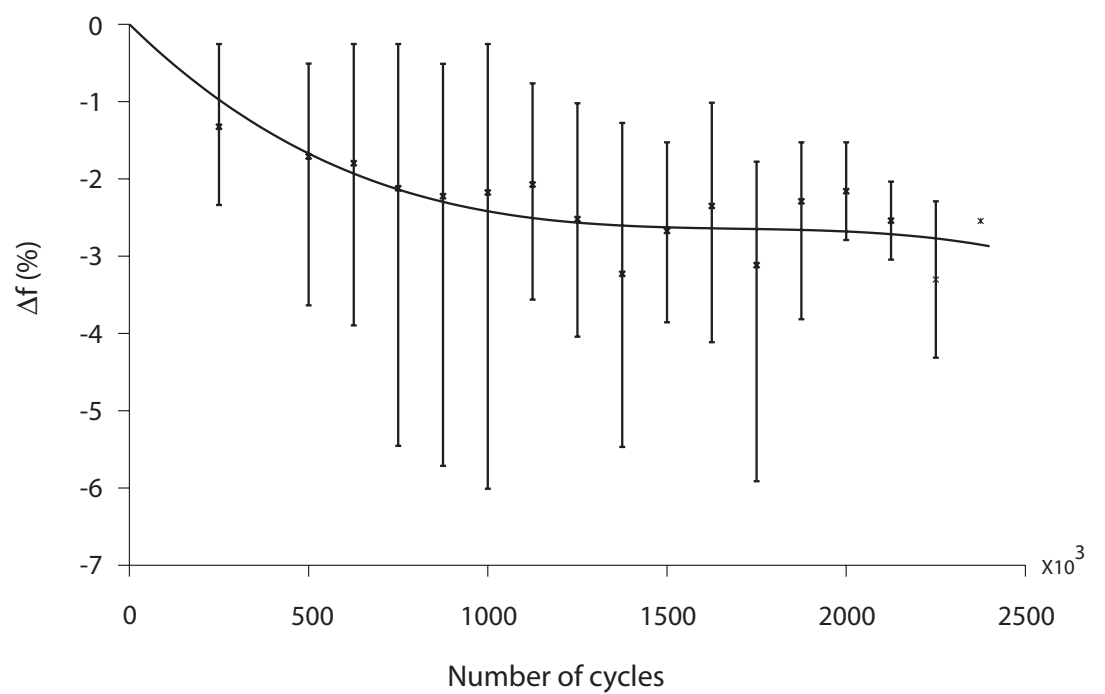

Figure 9: Normalized frequency variation versus number of cycles to failure. 


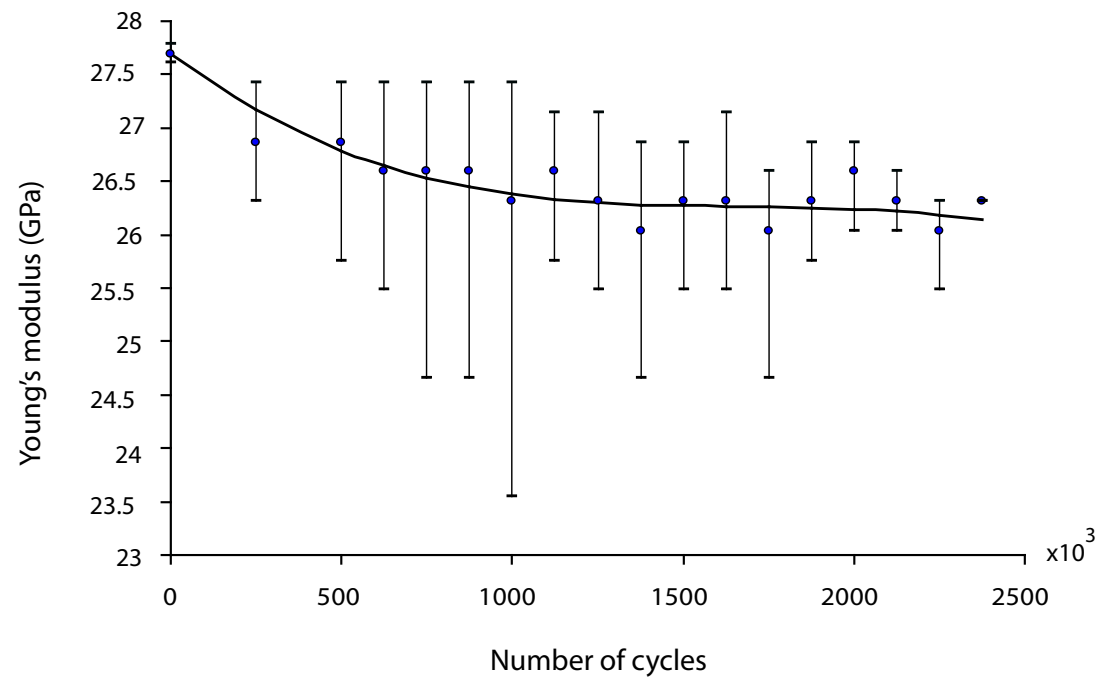

Figure 10: Young's modulus versus number of cycles to failure.

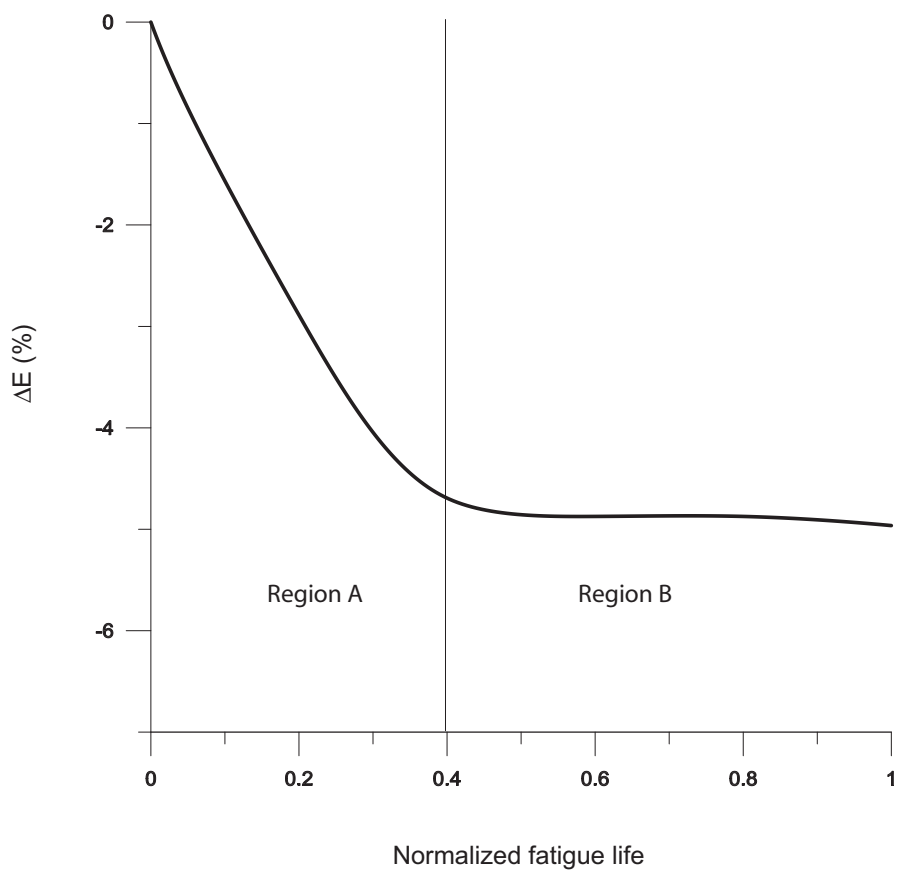

Figure 11: Normalized variation of elastic modulus versus normalized fatigue life. 
Table 1: Dynamic tests pattern.

\begin{tabular}{|c|c|c|c|}
\hline Percentage of Load & Mean Stress $(\mathrm{MPa})\left(\sigma_{i}^{m}\right)$ & Alternating Stress $(\mathrm{MPa})\left(\sigma_{i}^{a}\right)$ & Cycles $\left(\times 10^{6}\right)$ \\
\hline 50 & 1.61 & 1.23 & 0.25 \\
55 & 1.75 & 1.37 & 0.50 \\
60 & 1.90 & 1.52 & 0.75 \\
65 & 2.04 & 1.66 & 1.00 \\
70 & 2.18 & 1.80 & 1.25 \\
75 & 2.32 & 1.94 & 1.50 \\
80 & 2.46 & 2.09 & 1.75 \\
85 & 2.61 & 2.23 & 2.00 \\
90 & 2.75 & 2.37 & 2.25 \\
95 & 2.89 & 2.51 & 2.50 \\
100 & 3.03 & 2.65 & 2.75 \\
\hline
\end{tabular}

Table 2: Time required for other authors' conventional tests (References: ${ }^{(*)}$ E.Kholer [22])

\begin{tabular}{|c|c|c|c|}
\hline Specimen type & Frequency rate (hz) & Samples & Total time (hours) \\
\hline${\text { Concrete with cement Type I/II }{ }^{*}}^{*}$ & 12 & 10 & 643 \\
Concrete with cement Type III/A ${ }^{*}$ & 12 & 10 & 931 \\
Concrete with cement CSA-A ${ }^{*}$ & 12 & 10 & 531 \\
This work & 12 & 10 & 390 \\
\hline
\end{tabular}

